\title{
Two results on continuity and boundedness of stochastic convolutions
}

\author{
Stanisław Kwapieńn ${ }^{\mathrm{a}, 1}$, Michael B. Marcus ${ }^{\mathrm{b}, 2}$, Jan Rosiński ${ }^{\mathrm{c}, *, 3}$ \\ a Department of Mathematics, Warsaw University, Warsaw 02097, Poland \\ ${ }^{\mathrm{b}}$ Department of Mathematics, The City College of CUNY, New York, NY 10031, USA \\ c Department of Mathematics, University of Tennessee, Knoxville, TN 39996, USA
}

Received 3 January 2005; accepted 7 June 2005

Available online 7 December 2005

\section{Abstract}

Two results are obtained concerning the continuity and local boundedness of stochastic processes of the form

$$
(f * Z)(t)=\int_{0}^{t} f(t-s) \mathrm{d} Z(s), \quad t \geqslant 0,
$$

where $f:[0, \infty) \mapsto \mathbb{R}$ is a continuous function with $f(0)=0$ and $Z$ is a semimartingale. One states that the process $f * Z$ is not always continuous. Specifically, for any symmetric Lévy process $Z$ with paths of infinite variation there exists a function $f$, as above, such that $f * Z$ has locally unbounded paths almost surely.

The other states that the process $f * Z$ is continuous almost surely whenever $f$ is a sample path of any continuous Gaussian process with stationary increments that is independent of $Z$ and is equal to zero at zero.

() 2005 Elsevier SAS. All rights reserved.

\section{Résumé}

Pour les processus de la forme

$$
(f * Z)(t)=\int_{0}^{t} f(t-s) \mathrm{d} Z(s), \quad t \geqslant 0,
$$

où $f:[0, \infty) \mapsto \mathbb{R}$ est une fonction continue telle que $f(0)=0$ et où $Z$ est une semimartingale, deux résultats trajectoriels sont présentés. Le premier montre que le processus $f * Z$ n'est pas toujours continu. Plus précisément, pour tout processus de Lévy $Z$, symétrique et de variation infinie, il existe une fonction $f$ continue, avec $f(0)=0$, telle que $f * Z$ a presque sûrement des trajectoires localement non bornées. Le deuxième résultat montre que $f * Z$ est presque sûrement continu dés que $f$ est une trajectoire d'un processus gaussien continu à accroissements stationnaires, nulle à l'origine, qui est de plus indépendant de $Z$.

\footnotetext{
* Corresponding author.

E-mail addresses: kwapstan@mimuw.edu.pl (S. Kwapień), mbmarcus@optonline.net (M.B. Marcus), rosinski@ math.utk.edu (J. Rosiński).

1 Part of the research conducted during visit to the University of Tennessee, Knoxville. Supported in part by Polish Grant KBN $2 \mathrm{P} 03 \mathrm{~A} 02722$.

2 Research supported by grants from the National Science Foundation and PSCCUNY.

3 Research supported by a grant from the National Science Foundation.
} 
(c) 2005 Elsevier SAS. All rights reserved.

MSC: primary 60G17, 60G51, 60G15; secondary 60H05

Keywords: Stochastic convolution; Sample boundedness; Sample continuity; Semimartingales; Lévy processes

\section{Introduction}

In this paper we investigate the sample path continuity and boundedness of stochastic convolutions of the form

$$
(f * Z)(t):=\int_{0}^{t} f(t-s) \mathrm{d} Z(s), \quad t \in T:=[0,1],
$$

where $f: T \mapsto \mathbb{R}$ is a continuous function with $f(0)=0$ and $Z$ is a semimartingale. The continuity of $f * Z$ is obvious when $Z$ has paths of finite variation; in this case (1.1) is the usual Lebesgue-Stjeltjes integral with respect to each path of $Z$. The problem arises when the process $Z$ has paths of infinite variation.

When $Z$ is a Wiener process, $f * Z$ is a Gaussian process. The results of Fernique and Talagrand $[8,12]$ give necessary and sufficient conditions for the continuity of $f * Z$ in this case. When $Z$ is a Lévy process, $f * Z:=$ $\{(f * Z)(t), t \in T\}$ is an infinitely divisible process. A lot of attention has been given to the study of continuity properties of infinitely divisible processes, partly as a natural outgrowth of the study of Gaussian processes (see [15] and the references therein).

In [15] sufficient conditions are given for the continuity of general infinitely divisible processes. For processes of the form $f * Z$, these conditions depend on both $f$ and $Z$. Nevertheless, Theorem 2.5 [15] states that if $Z$ is a Lévy process and

$$
\sup _{u, v \in T}\left(\log \frac{1}{|u-v|}\right)^{1 / 2+\epsilon}|f(u)-f(v)|<\infty
$$

for some $\epsilon>0$, then $f * Z$ has a continuous version on $T$. Despite this result we are very far from understanding the continuity properties of (1.1) even in the seemingly straightforward case when $Z$ is a symmetric $\alpha$-stable process with $1 \leqslant \alpha<2$. The continuity of $f$ and $f(0)=0$ are simple necessary conditions for the continuity of (1.1) when $Z$ is a Lévy process with a nontrivial Poissonian part (but are not necessary when $Z$ is a Wiener process).

One fundamental point that eluded us was to show that (1.1) was not continuous for all bounded continuous functions $f$ with $f(0)=0$ whenever $Z$ is a Lévy process with paths of infinite variation. The first of the two main results of this paper, Theorem 3.1, shows this.

When $Z$ is a Wiener process, $f * Z$ is continuous almost surely whenever the Gaussian random Fourier series corresponding to $f$ is uniformly convergent almost surely (see the beginning of Section 3 ). The class of continuous one-periodic functions $f$, for which the corresponding Gaussian random Fourier series are continuous almost surely, forms the Pisier algebra, which is a proper subset of $C(T),[11,14,16]$. In [11] both sufficient and necessary conditions are given for a function $f$ to be in the Pisier algebra, expressed in terms of Fourier coefficients of $f$. However, there is no simple explicit characterization of the Pisier algebra.

When $Z$ is a pure jump Lévy process considering $f * Z$ as a random Fourier series is not helpful, as we explain in Section 3. Instead, we relate $f * Z$ to a Rademacher moving average process and construct an $f$ for which it is unbounded almost surely.

For general semimartingales $Z$, it is an open problem whether path continuity of $f * Z$, for every continuous $f$ with $f(0)=0$, implies that $Z$ has finite variation almost surely.

The continuity condition in (1.2) shows that any continuous function $f$, with $f(0)=0$, for which $f * Z$ is discontinuous must be very irregular. It is known that a continuous Gaussian process with stationary increments can have arbitrarily large moduli of continuity at all points in their domain (see, e.g., Theorem 2.4, [13]). Therefore, also from this point of view, it is interesting to consider convolution processes obtained by taking $f$ to be a sample path of a continuous Gaussian process with stationary increments, which is zero at zero. In Theorem 4.1 we prove that such processes are always continuous and we only require that $Z$ is a semimartingale. 
The results in Section 4 may have deeper content than simply showing that no smoothness condition on $f$ gives a necessary condition for the continuity of $f * Z$. By the relation $Z * G:=G * Z-Z(0) G$ one can define the stochastic convolution of a semimartingale with a stationary increment Gaussian process, such as fractional Brownian motion ( $Z$ and $G$ are independent). Thus Theorem 4.1 and Proposition 4.1 give us information about certain kind of stochastic integrals with respect to fractional Brownian motion.

It is interesting that the converse of Theorem 4.1 holds when $Z$ is a symmetric Lévy process (especially a Wiener process). In Proposition 4.2 we show that when $Z$ is a symmetric Lévy process and $G * Z$ is continuous, so is $G$. (The stationarity of the increments of $G$ is crucial in this result.)

There are many papers in the mathematical literature that consider convolutions like (1.1). The most recent one that we know of is [6]. Stochastic convolutions also occur naturally in applications, where $f$ is usually called the impulse response function and $Z$ is noise. An example in mathematical finance is the Heath-Jarrow-Morton-Musiela equation for the so called forward interest rate curve (see [9]). Another motivation for the study of processes $f * Z$ comes from certain problems in the theory of stochastic differential equations; see, e.g., [2], Chapter 6.

$C(T)$ stands for the Banach space of all continuous functions on $T$ with the usual supremum norm, denoted by $\|\cdot\|_{\infty}$. To avoid trivial complications we assume that all processes considered in this paper are separable.

\section{Two related stochastic convolutions}

We introduce another type of stochastic convolution that it is easier to work with. For a bounded measurable function $f:[-1,1] \mapsto \mathbb{R}$ and a semimartingale $Z$, we define

$$
(f \diamond Z)(t)=\int_{0}^{1} f(t-s) \mathrm{d} Z(s), \quad t \in T .
$$

Let $f \diamond Z:=\{(f \diamond Z)(t), t \in T\}$. The difference between (2.1) and $f * Z:=\{(f * Z)(t), t \in T\}$ is that the integration here is over the fixed interval $T$. The next lemma shows that the boundedness and continuity properties of the stochastic convolutions $f * Z$ and $f \diamond Z$ are closely related.

Lemma 2.1. Let $f$ be a continuous function on $[-1,1]$ with $f(0)=0$.

(a) Let $Z$ be an $L^{2}$ martingale. If $f \diamond Z$ has continuous paths almost surely and $E \sup _{t \in T}|(f \diamond Z)(t)|<\infty$, then $f * Z$ has continuous paths almost surely.

(b) Let $Z$ be a time-reversible semimartingale, i.e., $\{Z(1-)-Z((1-t)-), t \in T\} \stackrel{d}{=}\{Z(t)-Z(0), t \in T\}$ and, in addition, let $f$ be an even or odd function. If $f * Z$ has continuous (respectively bounded) paths almost surely then $f \diamond Z$ has continuous (respectively bounded) paths almost surely.

Proof. (a) Let $\left\{\mathcal{F}_{t}: t \in T\right\}$ be the filtration of $Z$ satisfying the standard assumptions, i.e., it is right continuous and $P$-complete. $f \diamond Z$ can be viewed as a random vector in the Banach space $C(T)$ that is 1-integrable in the Bochner sense. Consider the $C(T)$-valued conditional expectation process

$$
X_{t}=E\left(f \diamond Z \mid \mathcal{F}_{t}\right), \quad t \in T .
$$

$X=\left\{X_{t}, t \in T\right\}$ is a $L_{1}$-martingale in $C(T)$, hence its trajectories are càdlàg functions in $C(T)$ almost surely. (See Theorem 1, p. 181, [4].) For $u \in T$, let $e_{u}$ denote the linear functional on $C(T)$ which is the evaluation at $u$. Then

$$
e_{u}\left(X_{t}\right)=E\left((f \diamond Z)(u) \mid \mathcal{F}_{t}\right)=\int_{0}^{t} f(u-s) \mathrm{d} Z(s)
$$

almost surely. In particular, $(f * Z)(t)=e_{t}\left(X_{t}\right)$ almost surely, for each $t \in T$. Since a path $t \mapsto e_{t}\left(X_{t}\right)$ is càdlàg whenever $t \mapsto X_{t}$ is càdlàg, we infer that $f * Z$ has càdlàg paths and $(f * Z)(t)=e_{t}\left(X_{t}\right)$ almost surely, for all $t \in T$ (we assume separability of the processes under consideration). To prove that $f * Z$ is sample continuous, it is enough to show that for any stopping time $\tau$ with values in $T$

$$
\Delta_{\tau}(f * Z)=0
$$


almost surely, where for a càdlàg function $g$, we set $\Delta_{s} g:=g(s)-g(s-)$. (It is enough to consider $\tau$ of the form $\tau=\inf \left\{t>0:\left|\Delta_{t}(f * Z)\right|>\delta\right\} \wedge 1, \delta>0$.) Using (2.2) we get for each $u \in T$ and a stopping time $\tau$ in $T$

$$
e_{u}\left(\Delta_{\tau} X\right)=\Delta_{\tau}\left(e_{u}(X)\right)=f(u-\tau) \Delta_{\tau} Z
$$

almost surely, see, e.g., Theorem 13, p. 53, [17] or Theorem 10, p. 152, [4]. The above equation extends to $e_{\xi}\left(\Delta_{\tau} X\right)=$ $f(\xi-\tau) \Delta_{\tau} Z$, almost surely, for any simple random variable $\xi$ in the place of $u$, and then to arbitrary $\xi$ by the continuity of $e_{(\cdot)}$ and $f$. Taking $\xi=\tau$ we get

$$
\Delta_{\tau}(f * Z)=e_{\tau}\left(\Delta_{\tau} X\right)=f(\tau-\tau) \Delta_{\tau} Z=0
$$

almost surely because $f(0)=0$. Thus $f * Z$ has continuous paths almost surely.

To get (b) we write $f \diamond Z=f * Z+Y$ where $Y(t)=\int_{t}^{1} f(t-s) \mathrm{d} Z(s), t \in T$. From the time-reversibility of $Z$ we infer that the process $\widetilde{Y}(t):=\int_{1-t}^{1} f(t+s-1) \mathrm{d} Z(s), t \in T$, has the same distribution as $f * Z$. The proof is complete since $Y(t)=\epsilon \widetilde{Y}(1-t)$, where $\epsilon=-1$ when $f$ is odd and $\epsilon=1$ when $f$ is even.

Remark 2.1. The restrictions on $f$, that $f$ is continuous and $f(0)=0$ are necessary for the continuity of $f * Z$ whenever $Z$ is a Lévy process which is not a Wiener process; see [18].

Remark 2.2. If $Z$ is a symmetric Lévy process, then one can prove that for any continuous function $f$ on $[-1,1]$ with $f(0)=0$ and for every $c>0$,

$$
P\left(\sup _{t \in T}|(f * Z)(t)|>c\right) \leqslant 2 P\left(\sup _{t \in T}|(f \diamond Z)(t)|>c\right) .
$$

Obviously, part (b) of Lemma 2.1 applies when $Z$ is a Lévy process.

Remark 2.3. Under the assumptions of Lemma 2.1(a) we also have

$$
\sup _{c>0} c P\left(\sup _{t \in T}|(f * Z)(t)|>c\right) \leqslant E \sup _{t \in T}|(f \diamond Z)(t)| .
$$

Indeed, for every $t \in T$ we have

$$
|(f * Z)(t)|=\left|e_{t}\left(X_{t}\right)\right| \leqslant \sup _{u \in T}\left|e_{u}\left(X_{t}\right)\right|=\left\|E\left(f \diamond Z \mid \mathcal{F}_{t}\right)\right\|_{\infty} .
$$

Thus (2.3) follows from Doob's maximal inequality applied to the positive submartingale $\left\|E\left(f \diamond Z \mid \mathcal{F}_{t}\right)\right\|_{\infty}$.

\section{Unbounded stochastic convolutions}

Theorem 3.1. For each symmetric Lévy process $Z$ with paths of infinite variation, there exists a continuous oneperiodic function $f: \mathbb{R} \mapsto \mathbb{R}$, with $f(0)=0$, for which $f * Z$ in (1.1) has unbounded paths almost surely.

Before we proceed to the proof of Theorem 3.1 let us consider a simple approach which does not work for us but is instructive. Let $f$ be a continuous one-periodic function with Fourier series

$$
f(t)=\sum_{k=-\infty}^{\infty} a_{k} \mathrm{e}^{\mathrm{i} 2 \pi k t} .
$$

For each $t \in T$ we have

$$
(f \diamond Z)(t)=\int_{0}^{1} f(t-s) \mathrm{d} Z(s)=\sum_{k=-\infty}^{\infty} a_{k} \mathrm{e}^{\mathrm{i} 2 \pi k t} \int_{0}^{1} \mathrm{e}^{-\mathrm{i} 2 \pi k s} \mathrm{~d} Z(s)
$$

almost surely. If $Z$ is Wiener process, the stochastic integrals on the right-hand side of (3.1) are independent mean zero normal random variables. I.e., the right-hand side of (3.1) is a Gaussian random Fourier series. It has been known for some time that there are continuous functions $f$ for which the Gaussian process represented by the series 
in (3.1) is unbounded almost surely. The knowledge of this fact is assumed in Pisier's important paper [16] which characterizes the space of continuous complex-valued one-periodic functions $f$ for which the series (3.1) converges uniformly almost surely (the so-called Pisier's algebra). In Kahane's book, [11], page 218, Pisier's result is presented and a reference to other material in the book is given that shows how to find such functions. (Unfortunately, it seems to us, the reference in [11] is incomplete and should also mention Theorem 4, Chapter 5, in the book.) Kahane's observations are repeated, with credit, in a recent paper [1], which also elaborates on the nature of such functions. Using Theorem 1, Chapter 8 and Theorem 4, Chapter 5, [11] one can find a real-valued continuous one-periodic even (or odd) function $f$ with $f(0)=0$ for which (3.1) is unbounded almost surely. Then Lemma 2.1(b) together with a 0-1 law yield the conclusion of Theorem 3.1. Our approach in this case is similar to the one of [1]. In addition, Lemma 2.1(a) gives that $f * Z$ is continuous whenever $f$ belongs to the Pisier algebra.

This approach breaks down when $Z$ is a pure jump Lévy process. The stochastic integrals on the right-hand side of (3.1) are no longer independent. We really do not know anything about such series.

The next lemma is the main ingredient in the proof of Theorem 3.1.

Lemma 3.1. For every $\left\{a_{j}\right\} \in \ell_{2}$, with $\sum_{j}\left|a_{j}\right|=\infty$, there exists a continuous, even, 1-periodic function $g$ on $\mathbb{R}$ such that

$$
\|g\|:=\sup _{n} E\left\|\sum_{j=1}^{n} \epsilon_{j} a_{j} g\left(\cdot-U_{j}\right)\right\|_{\infty}=\infty .
$$

Here $\left\{\epsilon_{j}\right\}$ is a Rademacher sequence of independent random variables and $\left\{U_{j}\right\}$ is a sequence of independent random variables which are uniformly distributed in $[0,1]$ and which are independent of the sequence $\left\{\epsilon_{j}\right\}$.

Proof. Let $\mathcal{E}$ denote the Banach space of continuous, even, 1-periodic functions on $R$ with the sup norm. Suppose that the lemma is false, i.e. there exists a sequence $\left\{a_{j}\right\}$ as in the lemma such that $\|g\|<\infty$ for all $g \in \mathcal{E}$. Without loss of generality we assume that $a_{j}>0$ for all $j \geqslant 1$. Obviously $\|g\| \geqslant a_{1}\|g\|_{\infty}$. It is easy to check that this implies that $\|\cdot\|$ is a well defined complete norm on $\mathcal{E}$. Hence by the Closed Graph theorem the norm $\|\cdot\|$ is equivalent to the supremum norm. Thus for some constant $C$

$$
\|g\| \leqslant C\|g\|_{\infty} \quad \forall g \in \mathcal{E} .
$$

We show that for each $n$ there exists a function $g_{n} \in \mathcal{E}$ with $\left\|g_{n}\right\|_{\infty} \leqslant 1$ such that

$$
E\left\|\sum_{j=1}^{n} \epsilon_{j} a_{j} g_{n}\left(\cdot-U_{j}\right)\right\|_{\infty} \geqslant \frac{1}{2} \sum_{j=1}^{n} a_{j} .
$$

Since $\sum_{j=1}^{\infty} a_{j}=\infty$, this contradicts (3.3) and gives the proof.

For $k=1, \ldots, n$ we define $J_{k}$ to be the consecutive intervals of positive integers with each $J_{k}$ containing $k+2$ integers, i.e. $J_{k}=\{i \in N:(k-1)(k+4) / 2<i \leqslant k(k+5) / 2\}$. Denote each $t \in[0,1)$ by its dyadic expansion $t=\sum_{i=1}^{\infty} \delta_{i} 2^{-i}$, and whenever it is not unique, choose the finite one. Let $C_{k}$ be the subsets of $[0,1)$ defined by $C_{k}=\left\{t: t=\sum_{i=1}^{\infty} \delta_{i} 2^{-i}\right.$ with $\delta_{i}=0$ for $\left.i \in J_{k}\right\}$. Let $B_{n}=\bigcup_{k=1}^{n} C_{k}$.

Note that

$$
\left|B_{n}\right| \leqslant \sum_{k=1}^{n}\left|C_{k}\right|=\sum_{k=1}^{n} \frac{1}{2^{k+2}}<\frac{1}{4},
$$

where $|A|$ denotes the Lebesgue measure of $A \subset \mathbb{R}$. Let $B_{n}^{\prime}=B_{n} \cup\left(1-B_{n}\right)$. Let $g_{n}$ be a function in $\mathcal{E}$ such that, $\left\|g_{n}\right\|_{\infty}=1, \int_{0}^{1} g_{n}(t) \mathrm{d} t=0$ and $g_{n}(t)=1$ for $t \in B_{n}^{\prime}$. Such a function exists because the set $B_{n}^{\prime}$ is a finite union of intervals with total measure less than $\frac{1}{2}$. (Since $B_{n}^{\prime}$ is symmetric about $\frac{1}{2}, g_{n}$ can be chosen to be an even function.)

We claim that for every sequence of points $\left\{u_{k}\right\}_{k=1}^{n}$ there exists a $t \in[0,1)$ such that $\left(t-u_{k}\right)$ mod $1 \in C_{k} \subset B_{n}$ for each $k=1, \ldots, n$. Thus $g_{n}\left(t-u_{k}\right)=1$ for each $k=1, \ldots, n$. Consequently, for every sequence of positive numbers $\left\{b_{k}\right\}_{k=1}^{n}$, uniformly over the probability space,

$$
\left\|\sum_{k=1}^{n} b_{k} g_{n}\left(\cdot-U_{k}\right)\right\|_{\infty}=\sum_{k=1}^{n} b_{k} .
$$


Here is the proof of this claim. Observe that for every $k \geqslant 1$ and $x \in \mathbb{R}$ there exists an $s \in[0,1)$ with the dyadic expansion $s=\sum_{i \in J_{k}} \delta_{i} 2^{-i}$ such that $(s+x) \bmod 1 \in C_{k}$. Using this fact we construct recursively a sequence $t_{n}, \ldots, t_{1}$ with the property that $t_{k}=\sum_{i \in J_{k}} \delta_{i} 2^{-i}$ and $\left(t_{k}+\cdots+t_{n}-u_{k}\right) \bmod 1 \in C_{k}$, for $k=n, \ldots, 1$. Let $t=t_{1}+\cdots+t_{n}$. Note that for any $v \in C_{k},\left(t_{1}+\cdots+t_{k-1}+v\right) \bmod 1 \in C_{k}$. Since $\left(t_{k}+\cdots+t_{n}-u_{k}\right) \bmod 1 \in C_{k}$ it follows that $\left(t-u_{k}\right) \bmod 1 \in C_{k}$. This proves our claim and establishes (3.5)

For a particular fixed realization $\left\{\epsilon_{i}^{0}\right\}$ of the Rademacher sequence $\left\{\epsilon_{i}\right\}$, let $K=\left\{i: \epsilon_{i}^{0}=1, i \leqslant n\right\}, K^{\prime}=\left\{i: \epsilon_{i}^{0}=\right.$ $-1, i \leqslant n\}$ and $\mathcal{G}=\sigma\left(\left\{U_{i}\right\}_{i \in K}\right)$. Taking the expectation with respect to $\left\{U_{j}\right\}_{j=1}^{n}$ we see that

$$
\begin{gathered}
E\left\|\sum_{j=1}^{n} \epsilon_{j}^{0} a_{j} g_{n}\left(\cdot-U_{j}\right)\right\|_{\infty} \geqslant E\left\|\sum_{j \in K} a_{j} g_{n}\left(\cdot-U_{j}\right)-E\left(\sum_{j \in K^{\prime}} a_{j} g_{n}\left(\cdot-U_{j}\right) \mid \mathcal{G}\right)\right\|_{\infty} \\
=E\left\|\sum_{j \in K} a_{j} g_{n}\left(\cdot-U_{j}\right)\right\|_{\infty}=\sum_{j=1}^{n} a_{j} \frac{\epsilon_{j}^{0}+1}{2} .
\end{gathered}
$$

The last equality holds true by (3.5) and the preceding one because

$$
E\left(g_{n}\left(\cdot-U_{j}\right) \mid \mathcal{G}\right)=\int_{0}^{1} g_{n}(s) \mathrm{d} s=0
$$

for each $j \in K^{\prime}$. Now integrating with respect to the Rademacher sequence we obtain (3.4) which ends the proof of the lemma.

Proof of Theorem 3.1. By Theorem 1 [1] or by the discussion at the beginning of this section, Theorem 3.1 holds when $Z$ is a Wiener process. Therefore, it is enough to prove the theorem in the case when $Z$ is a pure jump Lévy process of infinite variation. Further, we can write $Z=Z_{0}+Z_{1}$, where $Z_{0}$ is a Lévy processes whose jumps magnitude do not exceed 1 and $Z_{1}$ is independent of $Z_{0}$ compound Poisson process. The process $f * Z_{1}$ is continuous for any continuous function $f$ with $f(0)=0$. Consequently, it is enough to prove the theorem in the case when $Z$ is a pure jump symmetric Lévy process of infinite variation whose jumps magnitude do not exceed 1 . Hence we may assume that

$$
E \mathrm{e}^{\mathrm{i} u Z(t)}=\exp \left\{t \int_{0}^{\infty}(\cos (u x)-1) \theta(\mathrm{d} x)\right\}
$$

where $\theta$ is a Lévy measure such that $\int_{0}^{1} x \theta(\mathrm{d} x)=\infty$ and $\theta((1, \infty))=0$. Using Proposition 2, [18] we can represent the processes $f \diamond Z$ by the almost surely convergent series

$$
(f \diamond Z)(t)=\sum_{j=1}^{\infty} \epsilon_{j} V_{j} f\left(t-U_{j}\right), \quad t \in T,
$$

where $\left\{\epsilon_{j}\right\}$ and $\left\{U_{j}\right\}$ are as in Lemma 3.1 and $V_{j}=\bar{\theta}^{-1}\left(\Gamma_{j}\right)$. Here $\left\{\Gamma_{j}\right\}$ is the sequence of arrival times in a Poisson process with the unit rate independent of $\left\{\epsilon_{j}, U_{j}\right\}$, and $\bar{\theta}^{-1}: \mathbb{R}_{+} \mapsto[0,1]$ is the right-continuous inverse of the function $\bar{\theta}(x)=\theta([x, \infty))$. For more information on this type of series see [19].

Let $a_{j}:=E V_{j}$. Then

$$
\sum_{j=1}^{\infty} a_{j}^{2} \leqslant \sum_{j=1}^{\infty} E V_{j}^{2}=\sum_{j=1}^{\infty} \int_{0}^{\infty} \bar{\theta}^{-1}(u)^{2} \frac{u^{j-1}}{j !} \mathrm{e}^{-u} \mathrm{~d} u=\int_{0}^{\infty} \bar{\theta}^{-1}(u)^{2} \mathrm{~d} u=\int_{0}^{1} x^{2} \theta(\mathrm{d} x)<\infty .
$$

Similarly,

$$
\sum_{j=1}^{\infty} a_{j}=\int_{0}^{1} x \theta(\mathrm{d} x)=\infty
$$


By Lemma 3.1 there exists a $g \in \mathcal{E}$ (i.e., a continuous, even, 1-periodic function) corresponding to the sequence $\left\{a_{j}\right\}$ such that $\|g\|=\infty$. Let $f(t)=g(t)-g(0)$. Then $f \in \mathcal{E}, f(0)=0$, and $\|f\|=\infty$. We will show that $f \diamond Z$ is unbounded almost surely. Assume, to the contrary, that it is bounded with a positive probability. Since $\Gamma_{j}$ 's are the partial sums of iid exponential random variables, the Hewitt-Savage 0-1 law applied to the series in (3.6) implies that $f \diamond Z$ is bounded almost surely. We will show that

$$
E\|f \diamond Z\|_{\infty}<\infty .
$$

Indeed, suppose that (3.7) does not hold. Then there exist sequences $\left\{t_{n}\right\} \subset T$ and $c_{n} \rightarrow 0$ such that

$$
E \sup _{n}\left|c_{n}(f \diamond Z)\left(t_{n}\right)\right|=\infty .
$$

Since $f \diamond Z$ is bounded almost surely, $\xi:=\left\{c_{n}(f \diamond Z)\left(t_{n}\right)\right\}_{n \geqslant 1}$ is an infinitely divisible random vector in the Banach space $c_{0}$. Its Lévy measure is the push-forward of the product of the Lebesgue measure on $[0,1]$ and $\tilde{\theta}$ by the map $(s, x) \mapsto\left\{c_{n} x f\left(t_{n}-s\right)\right\}_{n} \geqslant 1$, where $\tilde{\theta}$ is a symmetrization of $\theta$ on $R$. Thus $\xi$ has Lévy measure with bounded support, which implies that $E\|\xi\|_{c_{0}}<\infty$ (see, e.g., [3]). The latter condition contradicts (3.8) and so it proves (3.7).

Returning to (3.6) we notice that conditioned on $\left\{V_{j}\right\},\left\{\epsilon_{j} V_{j} f\left(\cdot-U_{j}\right)\right\}$ is a sequence of independent symmetric random vectors in $C(T)$. Therefore,

$$
\sup _{n} E\left\|\sum_{j=1}^{n} \epsilon_{j} V_{j} f\left(\cdot-U_{j}\right)\right\|_{\infty}=E\|f \diamond Z\|_{\infty}<\infty .
$$

By Fubini theorem

$$
\|f\|=\sup _{n} E\left\|\sum_{j=1}^{n} \epsilon_{j}\left(E V_{j}\right) f\left(\cdot-U_{j}\right)\right\|_{\infty} \leqslant \sup _{n} E\left\|\sum_{j=1}^{n} \epsilon_{j} V_{j} f\left(\cdot-U_{j}\right)\right\|_{\infty}<\infty
$$

which is a contradiction.

Thus $\{(f \diamond Z)(t), t \in T\}$ is unbounded almost surely and hence by Lemma 2.1(b), the process $\{(f * Z)(t), t \in T\}$ in (1.1) has unbounded paths with positive probability. Since the Lévy measure of $Z$ is infinite, the 0-1 law implies that $\{(f * Z)(t), t \in T\}$ is unbounded almost surely; see, e.g., Theorem 4.1, [20].

Remark 3.1. The above arguments and Lemma 3.1 show that under the assumptions of Lemma 3.1 there exists 1-periodic, even, continuous function $g$ such that the process

$$
Y(t)=\sum_{j=1}^{\infty} \epsilon_{j} a_{j} g\left(t-U_{j}\right), \quad t \in T,
$$

has unbounded paths almost surely.

\section{Convolutions of Gaussian processes with semimartingales}

We consider an interesting class of stochastic convolutions which are obtained by taking the impulse response function $f$ to be a sample path of a continuous Gaussian process with stationary increments which is equal to zero at zero. We show that for almost every selection of such an $f$, the resulting stochastic convolution process is continuous.

Theorem 4.1. Let $G=\{G(t), t \in \mathbb{R}\}$ be a continuous Gaussian process with stationary increments and $G(0)=0$ which is independent of a semimartingale $Z$. Then

$$
(G * Z)(t)=\int_{0}^{t} G(t-s) \mathrm{d} Z(s), \quad t \in T,
$$

is continuous almost surely. 
Before we proceed with the proof we recall some basic facts on Gaussian processes with stationary increments. Let $G$ be as given in the hypothesis; $E G(t)=m t$ for some constant $m$ by the stationarity of increments. $G$ can be represented by the real part of a complex, stationary increment process $G^{*}=\left\{G^{*}(t), t \in \mathbb{R}\right\}$ which admits the spectral representation

$$
G^{*}(t)=\int_{R}\left(\mathrm{e}^{\mathrm{i} t x}-1\right) W(\mathrm{~d} x)+W_{0} t+m(1+\mathrm{i}) t .
$$

Here $W$ is an independently scattered complex rotationally invariant Gaussian random measure on $\mathbb{R}$ with control measure $v(A)=E|W(A)|^{2}$ and with $E W(A)=0$ for all $A \in \mathcal{B}(\mathbb{R}), v$ is a symmetric Lévy measure on $\mathbb{R}$ and $W_{0}$ is a complex rotationally invariant normal random variable independent of $W$; see Chapter XI, Section 11, [5]. Since the real and imaginary parts of $G^{*}(t)$ are independent copies of each other it follows from (4.2) that

$$
E G(t) G(s)=\frac{1}{2} E G^{*}(t) \bar{G}^{*}(s)=\frac{1}{2} \int_{R}\left(1-\mathrm{e}^{\mathrm{i} t x}\right)\left(1-\mathrm{e}^{-\mathrm{i} s x}\right) v(\mathrm{~d} x)+c_{0} t s,
$$

where $c_{0}=E\left(\Re\left(W_{0}\right)\right)^{2}+m^{2}$. Hence

$$
\tau^{2}(t-s):=E|G(t)-G(s)|^{2}=\int_{R}(1-\cos (t-s) x) v(\mathrm{~d} x)+c_{0}(t-s)^{2},
$$

$\tau$ is a continuous even and nonnegative function on $\mathbb{R}$.

Let $\{X(t), t \in T\}$ be a mean zero Gaussian process with stationary increments and let $\rho^{2}(t, s)=\rho^{2}(t-s):=$ $E|X(t)-X(s)|^{2}$. Let $N(T, \rho, r)$ denote the minimum number of closed balls with centers in $T$ of radius $r$ (in the pseudo-metric $\rho$ ) that cover $T$. Set

$$
J(T, \rho)=\int_{0}^{\hat{\rho}}(\log (1+N(T, \rho, r)))^{1 / 2} \mathrm{~d} r
$$

where $\hat{\rho}:=\sup _{t, s \in T} \rho(t, s)$ is the diameter of $(T, \rho)$. By the Dudley-Fernique theorem, see, e.g., [8,12], we have that for some universal constants $C_{1}, C_{2}>0$

$$
C_{1} J(T, \rho) \leqslant E \sup _{t \in T}|X(t)| \leqslant E|X(0)|+C_{2} J(T, \rho) .
$$

There is a well known relation between the covering number $N$ for a translation invariant metric and Lebesgue measure

$$
\frac{|T|}{\left|\left\{t \in T^{\prime}: \rho(t) \leqslant r\right\}\right|} \leqslant N(T, \rho ; r) \leqslant \frac{\left|T^{\prime \prime}\right|}{\left|\left\{t \in T^{\prime}: \rho(t) \leqslant r / 2\right\}\right|},
$$

where $T^{\prime}=T-T=[-1,1]$ and $T^{\prime \prime}=T^{\prime}+T=[-1,2]$. (See [12], Lemma 13.1.) This allows us to estimate $J(T, \rho)$ as follows

$$
\frac{1}{3} J(T, \rho) \leqslant \int_{0}^{\hat{\rho}}\left(\log \left(1+\frac{1}{|\{t \in T: \rho(t) \leqslant r\}|}\right)\right)^{1 / 2} \mathrm{~d} r \leqslant 2 J(T, \rho) .
$$

To verify the first inequality in (4.7) we use the fact that $\psi(x)=(\log (1+x))^{1 / 2}$ is concave to get

$$
\begin{aligned}
J(T, \rho) & \leqslant \int_{0}^{\hat{\rho}} \psi\left(\frac{3}{2}\left|\left\{t \in T: \rho(t) \leqslant \frac{r}{2}\right\}\right|^{-1}\right) \mathrm{d} r \leqslant \frac{3}{2} \int_{0}^{\hat{\rho}} \psi\left(\left|\left\{t \in T: \rho(t) \leqslant \frac{r}{2}\right\}\right|^{-1}\right) \mathrm{d} r \\
& \leqslant 3 \int_{0}^{\hat{\rho}} \psi\left(|\{t \in T: \rho(t) \leqslant r\}|^{-1}\right) \mathrm{d} r .
\end{aligned}
$$

The second inequality in (4.7) follows similarly. 
Now we return to the continuous Gaussian process $G$ with stationary increments of Theorem 4.1. By the DudleyFernique theorem $G$ is continuous if and only if $J(T, \tau)<\infty$, or equivalently, by (4.7), if and only if

$$
\int_{0}^{\hat{\tau}}\left(\log \left(1+\frac{1}{|\{t \in T: \tau(t) \leqslant r\}|}\right)\right)^{1 / 2} \mathrm{~d} r<\infty,
$$

where $\tau$ is given by (4.4).

We need the following lemma for the proof of Theorem 4.1.

Lemma 4.1. Let $G$ be as given in Theorem 4.1 with $E G(t)=0$. Let $\left\{\Delta_{j}\right\}_{j=1}^{n}$ be a sequence of mean zero, uncorrelated random variables, independent of $G$, with $A:=\left(\sum_{j=1}^{n} E\left|\Delta_{j}\right|^{2}\right)^{1 / 2}$ and let $\left\{a_{j}\right\},\left\{b_{j}\right\}$ be two sequences of numbers with $\epsilon:=\max _{1 \leqslant j \leqslant n}\left|a_{j}-b_{j}\right|$. Consider

$$
H(t)=\sum_{j=1}^{n} \Delta_{j}\left(G\left(t-a_{j}\right)-G\left(t-b_{j}\right)\right), \quad t \in T .
$$

Then

$$
E \sup _{t \in T}|H(t)| \leqslant A(\hat{\tau}(\epsilon)+C L(\epsilon)),
$$

where $C$ is a universal constant, $\hat{\tau}(\epsilon)=\sup _{0 \leqslant t \leqslant \epsilon} \tau(t)$,

$$
K(\epsilon)=\left(\frac{1}{2} \int_{R} \min \left\{\epsilon^{2} x^{2}, 4\right\} v(\mathrm{~d} x)\right)^{1 / 2}
$$

and

$$
L(\epsilon)=\int_{0}^{K(\epsilon)}\left(\log \left(1+\frac{1}{|\{t \in T: \tau(t) \leqslant r\}|}\right)\right)^{1 / 2} \mathrm{~d} r .
$$

Proof. Suppose that $\left\{\Delta_{j}\right\}$ and $G$ are defined on a product probability space $\left(\Omega \times \Omega^{\prime}, \mathcal{F} \times \mathcal{F}^{\prime}, P \times P^{\prime}\right)$ such that $\Delta_{j}=\Delta_{j}(\omega)$ and $G=G\left(t, \omega^{\prime}\right),\left(\omega, \omega^{\prime}\right) \in \Omega \times \Omega^{\prime}$. Let $E_{G}$ denote the integral with respect to $P^{\prime}$. For each fixed $\omega \in \Omega$ the process $H_{\omega}$ defined on $\left(\Omega^{\prime}, \mathcal{F}^{\prime}, P^{\prime}\right)$ by

$$
H_{\omega}(t)=\sum_{j=1}^{n} \Delta_{j}(\omega)\left(G\left(t-a_{j}\right)-G\left(t-b_{j}\right)\right)
$$

is a Gaussian process with stationary increments and mean equal to zero.

Using (4.2) we get

$$
\begin{aligned}
\rho_{\omega}^{2}(t-s) & :=E_{G}\left|H_{\omega}(t)-H_{\omega}(s)\right|^{2} \\
& =\frac{1}{2} \int_{R}\left|\sum_{j=1}^{n} \Delta_{j}(\omega)\left(-\mathrm{e}^{\mathrm{i}\left(t-a_{j}\right) x}+\mathrm{e}^{\mathrm{i}\left(t-b_{j}\right) x}+\mathrm{e}^{\mathrm{i}\left(s-a_{j}\right) x}-\mathrm{e}^{\mathrm{i}\left(s-b_{j}\right) x}\right)\right|^{2} v(\mathrm{~d} x) \\
& =\frac{1}{2} \int_{R}\left|\sum_{j=1}^{n} \Delta_{j}(\omega)\left(\mathrm{e}^{\mathrm{i} t x}-\mathrm{e}^{\mathrm{i} s x}\right)\left(\mathrm{e}^{-\mathrm{i} b_{j} x}-\mathrm{e}^{-\mathrm{i} a_{j} x}\right)\right|^{2} v(\mathrm{~d} x) \\
& =\frac{1}{2} \int_{R}\left|\mathrm{e}^{\mathrm{i}(t-s) x}-1\right|^{2}\left|\sum_{j=1}^{n} \Delta_{j}(\omega)\left(\mathrm{e}^{-\mathrm{i} b_{j} x}-\mathrm{e}^{-\mathrm{i} a_{j} x}\right)\right|^{2} v(\mathrm{~d} x) .
\end{aligned}
$$

Taking the expectation and using the fact that 


$$
E\left|\sum_{j=1}^{n} \Delta_{j}\left(\mathrm{e}^{-\mathrm{i} b_{j} x}-\mathrm{e}^{-\mathrm{i} a_{j} x}\right)\right|^{2}=\sum_{j=1}^{n} E\left|\Delta_{j}\right|^{2}\left|\mathrm{e}^{\mathrm{i}\left(a_{j}-b_{j}\right) x}-1\right|^{2} \leqslant 4 A^{2}
$$

we get

$$
(E \rho(t-s))^{2} \leqslant E \rho^{2}(t-s) \leqslant 4 A^{2} \tau^{2}(t-s) .
$$

That is,

$$
E \rho(t) \leqslant 2 A \tau(t), \quad t \in T .
$$

Because $\left|\mathrm{e}^{\mathrm{i} u}-1\right| \leqslant \min \{|u|, 2\}$, for each $u \in \mathbb{R}$, we see from (4.10) that

$$
\hat{\rho}_{\omega}^{2}:=\sup _{t \in T} \rho_{\omega}^{2}(t) \leqslant \frac{1}{2} \int_{R} \min \left\{x^{2}, 4\right\}\left|\sum_{j=1}^{n} \Delta_{j}(\omega)\left(\mathrm{e}^{-\mathrm{i} b_{j} x}-\mathrm{e}^{-\mathrm{i} a_{j} x}\right)\right|^{2} v(\mathrm{~d} x) .
$$

Hence

$$
E \hat{\rho}^{2} \leqslant \frac{1}{2} \int_{R} \min \left\{x^{2}, 4\right\} \sum_{j=1}^{n} E \Delta_{j}^{2}\left|\mathrm{e}^{\mathrm{i}\left(a_{j}-b_{j}\right) x}-1\right|^{2} v(\mathrm{~d} x) \leqslant 2 A^{2} \int_{R} \min \left\{\epsilon^{2} x^{2}, 4\right\} \nu(\mathrm{d} x)=4 A^{2} K^{2}(\epsilon) .
$$

By (4.6) and (4.7)

$$
\begin{aligned}
E_{G} \sup _{t \in T}\left|H_{\omega}(t)\right| & \leqslant E_{G}\left|H_{\omega}(0)\right|+C E_{G} J\left(T, \rho_{\omega}\right) \\
& \leqslant E_{G}\left|H_{\omega}(0)\right|+3 C E_{G} \int_{0}^{\hat{\rho}_{\omega}}\left(\log \left(1+\frac{1}{\left|\left\{t \in T: \rho_{\omega}(t) \leqslant r\right\}\right|}\right)\right)^{1 / 2} \mathrm{~d} r .
\end{aligned}
$$

Applying the remaining expectation and using Proposition 1.4.2, [7], and (4.11)-(4.13) we get

$$
\begin{aligned}
E \sup _{t \in T}|H(t)| & \leqslant E|H(0)|+3 C E \int_{0}^{\hat{\rho}}\left(\log \left(1+\frac{1}{|\{t \in T: \rho(t) \leqslant r\}|}\right)\right)^{1 / 2} \mathrm{~d} r \\
& \leqslant E|H(0)|+3 C \int_{0}^{E \hat{\rho}}\left(\log \left(1+\frac{1}{|\{t \in T: E \rho(t) \leqslant r\}|}\right)\right)^{1 / 2} \mathrm{~d} r \\
& \leqslant E|H(0)|+3 C \int_{0}^{2 A K(\epsilon)}\left(\log \left(1+\frac{1}{\mid\{t \in T: \tau(t) \leqslant r /(2 A) \mid}\right)\right)^{1 / 2} \mathrm{~d} r \\
& \leqslant A(\hat{\tau}(\epsilon)+6 C L(\epsilon)) .
\end{aligned}
$$

The last bound follows from the fact that

$$
(E|H(0)|)^{2} \leqslant E H^{2}(0)=\sum_{j=1}^{n} E \Delta_{j}^{2} \tau^{2}\left(a_{j}-b_{j}\right) .
$$

This completes the proof of Lemma 4.1 .

Proof of Theorem 4.1. Since $G$ has stationary increments $E G(t)=m t$ for some constant $m$. Theorem 4.1 holds when $G(t)$ is replaced by $m t$, since $\int_{0}^{t}(t-s) \mathrm{d} Z(s)=\int_{0}^{t} Z(s) \mathrm{d} s$. Therefore, we can assume that $E G(t)=0$ for all $t \in \mathbb{R}$. 
As we point out in Section 1, the process $G * Z$ is continuous if $Z$ has paths of bounded variation on finite intervals, almost surely. By the standard localization procedure we can assume that $Z$ is a square integrable martingale with $A=\left(E Z^{2}(1)\right)^{1 / 2}$. Finally, by Lemma 2.1 (a) it is enough to prove the continuity of the process

$$
(G \diamond Z)(t)=\int_{0}^{1} G(t-s) \mathrm{d} Z(s), \quad t \in T,
$$

and the integrability of its sup norm.

Let $\left\{\pi_{n}\right\}:=\left\{0=t_{0}^{n}<t_{1}^{n}<\cdots<t_{k_{n}}^{n}=1\right\}$ be a normal and nested sequence of partitions of $T$. Put $\epsilon_{n}:=$ $\max _{1 \leqslant i \leqslant k_{n}}\left(t_{i}^{n}-t_{i-1}^{n}\right)$ and $\Delta_{i}^{n}=Z\left(t_{i}^{n}\right)-Z\left(t_{i-1}^{n}\right), n \geqslant 1, i=1, \ldots, k_{n}$. Let

$$
X^{(n)}(t)=\sum_{i=1}^{k_{n}} G\left(t-t_{i-1}^{n}\right) \Delta_{i}^{n}, \quad t \in T .
$$

Clearly $\left\{X^{(n)}(t), t \in T\right\}$ has continuous paths. For $m>n$ consider

$$
H^{n, m}(t):=X^{(n)}(t)-X^{(m)}(t)=\sum_{i=1}^{k_{n}} \sum_{\left\{j: t_{i-1}^{n}<t_{j}^{m} \leqslant t_{i}^{n}\right\}}\left(G\left(t-t_{i-1}^{n}\right)-G\left(t-t_{j-1}^{m}\right)\right) \Delta_{j}^{m} .
$$

Applying Lemma 4.1 we get

$$
E \sup _{t \in T}\left|H^{n, m}(t)\right| \leqslant A\left(\hat{\tau}\left(\epsilon_{n}\right)+C L\left(\epsilon_{n}\right)\right) .
$$

Since the right side of the inequality goes to 0 when $n \rightarrow \infty$, we conclude that the processes $\left\{X^{(n)}(t), t \in T\right\}$ converge uniformly in $L_{1}$ to a continuous process $\left\{X^{(\infty)}(t), t \in T\right\}$. By the definition of the stochastic integral, $X^{(\infty)}(t)=$ $(G \diamond Z)(t)$ almost surely, for each $t \in T$. This proves Theorem 4.1 .

Lemma 4.1 and the proof of Theorem 4.1 easily yield the following estimate which we believe is of some interest

Proposition 4.1. If $Z$ is an $L^{2}$-martingale and $G$ is as in Theorem 4.1, then

$$
\sup _{c>0} c P\left(\sup _{t \in T}|(G * Z)(t)|>c\right) \leqslant C\left(E \sup _{t \in T} G^{2}(t)\right)^{1 / 2}\left(E Z^{2}(1)\right)^{1 / 2},
$$

for some universal constant $C$.

Proof. As in the proof of Theorem 4.1 we may assume that $E G(t)=0$. Keeping the notation of Lemma 4.1 we estimate $K(1)$ and $L(1)$. By (4.3) it follows that

$$
K^{2}(1)=\frac{1}{2} \int_{R} \min \left\{x^{2}, 4\right\} v(\mathrm{~d} x) \leqslant 8 \int_{R}\left(\int_{0}^{1}\left|\mathrm{e}^{\mathrm{i} x t}-1\right|^{2} \mathrm{~d} t\right) v(\mathrm{~d} x) \leqslant 16 \sup _{t \in T} E G^{2}(t) .
$$

By (4.6), (4.7) and because $|\{t \in T: \tau(t) \leqslant r\}|=1$ for $r>\hat{\tau}$, we get

$$
\begin{aligned}
L(1) & =\int_{0}^{\hat{\tau}}\left(\log \left(1+\frac{1}{|\{t \in T: \tau(t) \leqslant r\}|}\right)\right)^{1 / 2} \mathrm{~d} r+\int_{\hat{\tau}}^{K(1)}(\log 2)^{1 / 2} \mathrm{~d} r \\
& \leqslant C_{1}\left(E \sup _{t \in T} G^{2}(t)\right)^{1 / 2}+K(1)(\ln 2)^{1 / 2} \leqslant C_{2}\left(E \sup _{t \in T} G^{2}(t)\right)^{1 / 2},
\end{aligned}
$$

where $C_{1}$ and $C_{2}$ are universal constants. Since $\hat{\tau}=\sup _{t \in T}\left(E G^{2}(t)\right)^{1 / 2}$ and since for each $L_{2}$-martingale $Z$, $A \leqslant\left(E Z^{2}(1)\right)^{1 / 2}$, the above estimate of $L(1)$ and Lemma 4.1 imply that for some universal constant $C_{3}$

$$
E \sup _{t \in T}\left|X^{n}(t)-X^{0}(t)\right| \leqslant C_{3}\left(\sup _{t \in T} E G^{2}(t)\right)^{1 / 2}\left(E Z^{2}(1)\right)^{1 / 2},
$$


where $X^{n}$ are the processes defined in the proof of Theorem 4.1. We choose $\pi_{0}=\{0,1\}$ which gives $X^{0}(t)=$ $G(t)(Z(1)-Z(0))$. As shown in the proof of that theorem, $\lim _{n \rightarrow \infty} E \sup _{t \in T}\left|X^{n}(t)-G \diamond Z(t)\right|=0$. Therefore, for a universal constant $C$, we have

$$
E \sup _{t \in T}|G \diamond Z(t)| \leqslant \lim _{n \rightarrow \infty} E \sup _{t \in T}\left|X^{n}(t)-X^{0}(t)\right|+E \sup _{t \in T}\left|X^{0}(t)\right| \leqslant C\left(\sup _{t \in T} E G^{2}(t)\right)^{1 / 2}\left(E Z^{2}(1)\right)^{1 / 2} .
$$

This and the inequality (2.3) conclude the proof of Proposition 4.1.

Remark 4.1. If $Z$ is a symmetric Lévy process with $E Z^{2}(1)<\infty$, then using the result stated in Remark 2.2 we obtain that

$$
E \sup _{t \in T}|(G * Z)(t)| \leqslant C\left(E \sup _{t \in T} G^{2}(t)\right)^{1 / 2}\left(E Z^{2}(1)\right)^{1 / 2},
$$

where $C$ is a universal constant.

If $Z$ is a symmetric Lévy process we have the following converse to Theorem 4.1.

Proposition 4.2. Let $Z$ be a symmetric Lévy process and let $G$ be a Gaussian process with stationary increments, continuous in probability, with $G(0)=0$, and independent of $Z$. If the process $G * Z$ is bounded almost surely then the process $G$ is continuous almost surely.

Proof. Note that because $G$ has stationary increments it is either continuous almost surely or else unbounded on all finite intervals almost surely. (See Chapter III, Section 4, [10].) As in the proof of Theorem 4.1, we may assume that $G$ has mean zero. If $Z$ is not a Wiener process, then it contains an independent symmetric compound Poisson factor, say $Z_{p}$. By symmetry, the process $G * Z_{p}$ is bounded almost surely. This trivially implies that $G$ is bounded, hence continuous almost surely. Therefore, we may only consider the case when $Z$ is a Wiener process. Suppose that $Z$ and $G$ are defined on a product probability space $\left(\Omega \times \Omega^{\prime}, \mathcal{F} \times \mathcal{F}^{\prime}, P \times P^{\prime}\right)$ such that $Z_{t}=Z_{t}(\omega)$ and $G=G\left(t, \omega^{\prime}\right)$, $\left(\omega, \omega^{\prime}\right) \in \Omega \times \Omega^{\prime}$.

Let $Z$ be a standard Wiener process and assume that $G * Z$ is bounded on $T$ almost surely. Without loss of generality we may also assume that $Z$ and $G$ are rotationally invariant complex valued processes (thus $E|Z(1)|^{2}=2$ ). By (4.2) (with $m=0$ ) we have

$$
G(t)=G_{0}(t)+G_{1}(t)+W_{0} t+W_{1},
$$

where

$$
\begin{aligned}
& G_{0}(t)=\int_{|x| \leqslant 1}\left(\mathrm{e}^{\mathrm{i} t x}-1\right) W(\mathrm{~d} x), \\
& G_{1}(t)=\int_{|x|>1} \mathrm{e}^{\mathrm{i} t x} W(\mathrm{~d} x),
\end{aligned}
$$

and

$$
W_{1}=-W\left([-1,1]^{c}\right) .
$$

The process $G_{0}$ has absolutely continuous sample paths almost surely and its derivative is given by

$$
G_{0}^{\prime}(t)=\mathrm{i} \int_{|x| \leqslant 1} \mathrm{e}^{\mathrm{i} t x} x W(\mathrm{~d} x) .
$$

Indeed, since

$$
E \int_{0}^{1}\left|G_{0}^{\prime}(t)\right|^{2} \mathrm{~d} t=\int_{0|x| \leqslant 1}^{1} \int_{|x| \leqslant 1} x^{2} v(\mathrm{~d} x) \mathrm{d} t=\int_{|x|} x^{2} v(\mathrm{~d} x)<\infty
$$


process $G_{0}^{\prime}$ has square integrable trajectories almost surely and $G_{0}(t)=\int_{0}^{t} G_{0}^{\prime}(s) \mathrm{d} s$. Hence $G_{0} * Z=Z * G_{0}$ has continuous paths almost surely. By (4.17) and our assumption $G_{1} * Z$ is bounded almost surely and it is enough to show that the stationary process $G_{1}$ is continuous almost surely.

By Lemma 2.1(b), $G_{1} \diamond Z$ is bounded almost surely. Since $G_{1} \diamond Z$ is a second order Gaussian chaos process, $E \sup _{t \in T}\left|\left(G_{1} \diamond Z\right)(t)\right|<\infty$. We have

$$
\left(G_{1} \diamond Z\right)(t)=\int_{|x|>1} \mathrm{e}^{\mathrm{i} t x} \int_{0}^{1} \mathrm{e}^{-\mathrm{i} s x} \mathrm{~d} Z(s) W(\mathrm{~d} x)=: \int_{|x|>1} \mathrm{e}^{\mathrm{i} t x} Y(x) W(\mathrm{~d} x) .
$$

By checking their covariance functions, conditionally for a fixed realization of $Z=Z(\cdot, \omega)$, we see that the Gaussian processes

$$
\left\{\int_{|x|>1} \mathrm{e}^{\mathrm{i} t x}|Y(x, \omega)| W(\mathrm{~d} x), t \in R\right\}
$$

and

$$
\left\{\int_{|x|>1} \mathrm{e}^{\mathrm{i} t x} Y(x, \omega) W(\mathrm{~d} x), t \in R\right\}
$$

have the same distribution. Hence

$$
\begin{aligned}
E \sup _{t \in T}\left|\left(G_{1} \diamond Z\right)(t)\right| & =E_{Z} E_{G} \sup _{t \in T}\left|\int_{|x|>1} \mathrm{e}^{\mathrm{i} t x} Y(x, \omega) W(\mathrm{~d} x)\right|=E_{Z} E_{G} \sup _{t \in T}\left|\int_{|x|>1} \mathrm{e}^{\mathrm{i} t x}\right| Y(x, \omega)|W(\mathrm{~d} x)| \\
& \geqslant E_{G} \sup _{t \in T}\left|\int_{|x|>1} \mathrm{e}^{\mathrm{i} t x} E_{Z}\right| Y(x)|W(\mathrm{~d} x)|=\sqrt{\frac{\pi}{2}} E_{G} \sup _{t \in T}\left|\int_{|x|>1} \mathrm{e}^{\mathrm{i} t x} W(\mathrm{~d} x)\right| \\
& \geqslant \sqrt{\frac{\pi}{2}} E \sup _{t \in T}\left|G_{1}(t)\right| .
\end{aligned}
$$

The next to last equation follows from the fact that for each $x, Y(x)$ is the standard complex valued normal random variable, and so $E|Y(x)|=\sqrt{\frac{\pi}{2}}$. Since we are assuming that the first term in (4.18) is bounded, we get that $G_{1}$ is bounded and thus continuous. The proof is complete.

\section{Acknowledgement}

The authors are grateful to the referee for his careful reading of the paper and his helpful suggestions.

\section{References}

[1] Z. Brzeźniak, S. Peszat, J. Zabczyk, Continuity of stochastic convolutions, Czechoslovak Math. J. 51 (2001) 679-684.

[2] G. Da Prato, J. Zabczyk, Stochastic Equations in Infinite Dimensions, Encyclopedia Math. Appl., vol. 45, Cambridge Univ. Press, Cambridge, 1992.

[3] A. de Acosta, Exponential moments of vector valued random series and triangular arrays, Ann. Probab. 8 (1980) 381-389.

[4] N. Dinculeanu, Vector Integration and Stochastic Integration in Banach Spaces, Willey \& Sons, Inc., 2000.

[5] J.L. Doob, Stochastic Processes, J. Willey \& Sons, Inc., New York, Chapman \& Hall, Londres, 1953.

[6] M. Errami, F. Russo, Covariation de convolution de martingales, C. R. Acad. Sci. Paris Sér. I Math. 326 (5) (1998) $601-606$.

[7] X. Fernique, Continuité et théorème central limite pour les transformées de Fourier des mesures aléatoires du second ordre, Wahr. Verw. Gebiete 42 (1978) 57-66.

[8] X. Fernique, Fonctions aléatoires gaussiennes, vecteurs aléatoires gaussiens, Université de Montréal, Centre de Recherches Mathématiques, Montreal, 1997.

[9] D. Heath, A. Jarrow, A. Morton, Bond pricing and the term structure of interest rate: a new methodology for contingent claim valuation, Econometrica 60 (1992) 77-105.

[10] N. Jain, M.B. Marcus, Continuity of subgaussian processes, in: Probability on Banach Spaces, in: Adv. Probab., vol. 4, Marcel Dekker, New York, 1978, pp. 81-196. 
[11] J.P. Kahane, Some Random Series of Functions, second ed., Cambridge Univ. Press, Cambridge, 1985.

[12] M. Ledoux, M. Talagrand, Probability in Banach Spaces. Isoperimetry and Processes, Springer-Verlag, New York, 1991.

[13] M.B. Marcus, Gaussian lacunary series and the modulus of continuity for Gaussian processes, Z. Wahr. Verw. Gebiete 22 (1972) $301-322$.

[14] M.B. Marcus, G. Pisier, Random Fourier Series with Applications to Harmonic Analysis, Princeton Univ. Press, 1981.

[15] M.B. Marcus, J. Rosiński, Continuity and boundedness of infinitely divisible processes: a Poisson point process approach, J. Theoret. Probab. 18 (2005) 109-160.

[16] G. Pisier, A remarkable homogeneous Banach algebra, Israel J. Math. 34 (1979) 38-44.

[17] P. Protter, Stochastic Integration and Differential Equations, Springer-Verlag, New York, 1990.

[18] J. Rosiński, On path properties of certain infinitely divisible processes, Stochastic Process. Appl. 33 (1989) $73-87$.

[19] J. Rosiński, Series representations of Lévy processes from the perspective of point processes, in: O.E. Barndorff-Nielsen, T. Mikosch, S.I. Resnick (Eds.), Lévy Processes - Theory and Applications, Birkhäuser, Boston, 2001, pp. 401-415.

[20] J. Rosiński, G. Samorodnitsky, Symmetrization and concentration inequalities for multilinear forms with applications to zero-one laws for Lévy chaos, Ann. Probab. 24 (1996) 422-437. 